\title{
Revisión pictográfica de endoleaks (endofugas)
}

\author{
Dres. Pablo Alvayay $Q^{(1)}$, Giancarlo Schiappacasse $F^{(2)}$, Andrés Labra $W^{(2)}$, Cristián Sakamoto $G^{(1)}$, Cristobal Ramos $G^{(2)}$.
}

1. Residente Postgrado Programa de Especialidad en Radiología, Facultad de Medicina Clínica Alemana, Universidad del Desarrollo. Santiago - Chile.

2. Médico Radiólogo, Facultad de Medicina Clínica Alemana, Universidad del Desarrollo. Santiago - Chile.

\section{A Pictorial review of endoleaks}

Abstract: The current treatment for aortic aneurysms is to install an endovascular stent in the aortic lumen. The most common complication of stents is endoleaks. Those defined as a peri-prosthetic vascular leak, in the aneurysm sac, are usually asymptomatic. If not detected early, they can progress with the growth and rupture of the aneurysm. The method of choice for evaluation is angiography by computed tomography (CT). The aim of this pictorial review is to describe and illustrate the imaging findings of the different types of endoleaks in computed tomography angiograms (5 types).

Keywords: Aorta, CT angiography, Endoleak, Stents.

Resumen: El tratamiento actual de los aneurismas aórticos es la instalación de una endoprótesis en el lumen aórtico por vía endovascular. La complicación más frecuente de las endoprótesis son los endoleaks. Los que se definen como flujo vascular peri-protésico, en el saco aneurismático, generalmente asintomático. De no ser detectados a tiempo, pueden progresar con el crecimiento y rotura del aneurisma. El método de elección para su evaluación es la angiografía mediante tomografía computada (TC).

El objetivo de la presente revisión pictográfica es describir e ilustrar los hallazgos imaginológicos de los diferentes tipos de endoleaks en angiografía por tomografía computada (cinco tipos).

Palabras clave: Angiografía por CT, Aorta, Endofuga, Endoprótesis.

Alvayay P, Schiappacasse G, Labra A, Sakamoto C, Ramos C. Revisión pictográfica de endoleaks (endofugas). Rev Chil Radiol 2015; 21(2): 66-69.

Correspondencia: Dr. Cristian Sakamoto G. / csakamotog@gmail.com

Trabajo recibido el 18 de mayo de 2015. Aceptado para publicación el 06 de julio de 2015.

\section{Introducción}

El tratamiento de elección de los aneurismas aórticos es la instalación de una endoprótesis en el lumen aórtico por vía endovascular, logrando dirigir el flujo a través del aneurisma, previniendo el crecimiento y/o rotura de éste ${ }^{(1,2)}$.

Las endoprótesis no están exentas de complicaciones. Dentro de las más frecuentes podemos encontrar: endoleaks o endofugas ${ }^{(1-4)}$, trombosis de la endoprótesis ${ }^{(2,4)}$, angulación de la endoprótesis ${ }^{(2)}$, migración de la endoprótesis desde el sitio de fijación ${ }^{(2,3)}$ y formación de pseudoaneurismas secundarios a una lesión de la pared arterial ${ }^{(2,3)}$.

Los endoleaks son la complicación más frecuente, con una frecuencia estimada entre 15 a $52 \%{ }^{(5)}$, y de no ser detectados a tiempo, pueden progresar mediante el crecimiento y rotura del aneurisma. Es por esto que se hace necesario conocer y clasificar adecuadamente estas complicaciones, orientando el manejo y tratamiento adecuados.

Los endoleaks se definen como flujo vascular peri-protésico, en el saco aneurismático, generalmente asintomático y que no suelen generan complicaciones hasta etapas avanzadas. Es necesario un control periódico mediante imágenes de todas las endoprótesis, para descartar la aparición de estas complicaciones.

El método de elección para su evaluación inicial es la angiografía mediante tomografía computada (TC) con fases no contrastada, arterial y tardía, con una sensibilidad de $94 \%$ y especificidad de $93 \%{ }^{(6)}$. 
Debido a que estos pacientes requieren control de por vida, es crítico reducir la dosis de radiación a la menor posible. Algunos autores han optado por utilizar sólo las fases sin contraste y tardía, manteniendo una sensibilidad diagnóstica adecuada ${ }^{(7)}$. Otros autores como Lezzi et al. proponen el seguimiento posterior sólo con fase arterial, ya que permite detectar complicaciones clínicamente significativas y que requerirán eventual tratamiento activo(5). Por su parte las complicaciones detectadas en fases tardías no suelen tener repercusión clínica relevante para el manejo del paciente, ya que al ser de bajo flujo, evolucionan de manera auto-limitada.

Otros métodos de imágenes para la valoración no invasiva son la resonancia magnética y el ultrasonido con Doppler, han reportado rendimientos inferiores para la detección y caracterización de endofugas, y no serán tratados en esta revisión.

\section{Desarrollo}

Debido a que los endoleaks son las complicaciones más frecuentes, es importante conocer las características y el tratamiento posterior de estas complicaciones.

Para optimizar el manejo de estas, Veith et al. ${ }^{(8)}$ clasificó los endoleaks de acuerdo al origen del flujo sanguíneo en cinco tipos:

- Endoleaks tipo I, se definen como la presencia de flujo sanguíneo fuera del lumen protésico, adyacente al sitio de anclaje proximal (IA) (Figura 1) o distal (IB) (Figura 2) en la arteria. Se producen por un sello insuficiente entre la prótesis y la pared arterial, siendo más comunes en las endoprótesis torácicas. Se deben detectar precozmente, ya que requieren reparación inmediata ${ }^{(3,4,9,10)}$.

- Endoleak tipo Il, es el tipo más común, se produce por flujo retrógrado desde una rama arterial excluida por la endoprótesis (más frecuentemente de las arterias mesentérica inferior y lumbares, también se produce desde las arterias intercostales y renal accesoria) (Figura 3). En la fase tardía de la angiografía por TC

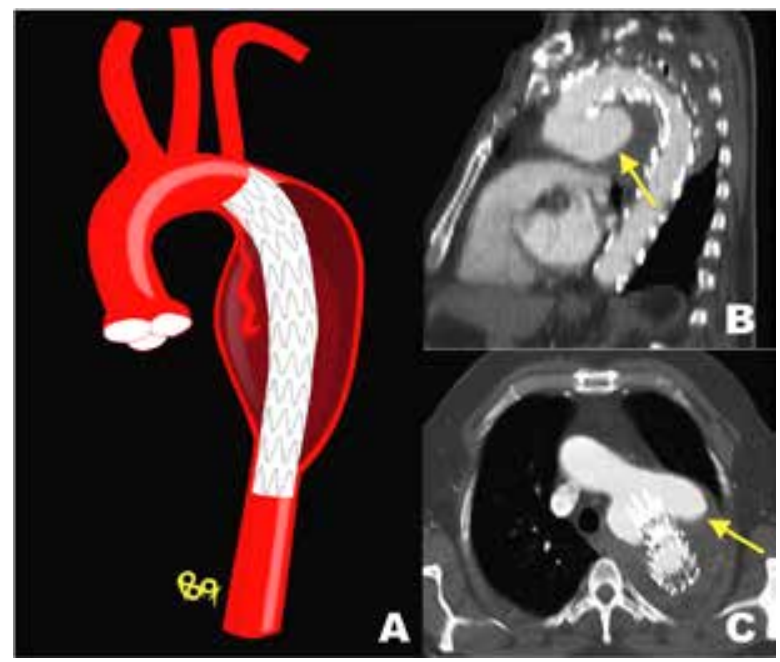

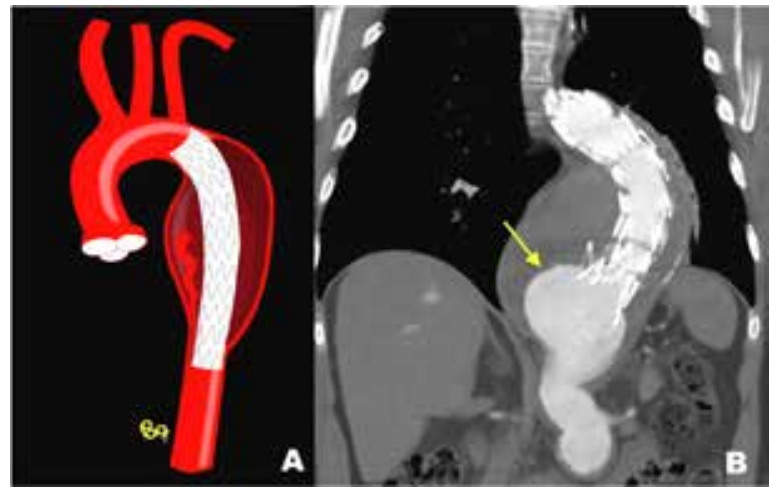

Figura 2. Endoleak tipo IB. a) Esquema representativo. b) Reconstrucción coronal de TC de tórax contrastada de paciente con aneurisma de la aorta tóraco-abdominal que presenta salida de medio de contraste (flecha) desde el extremo distal de la prótesis.

se identifica flujo de contraste en la periferia del saco aneurismático, que no suele contactar directamente con la endoprótesis ${ }^{(3,4,9-11)}$. El manejo de este tipo de fuga es controversial, se puede realizar seguimiento hasta su resolución espontánea o embolizar la arteria excluida en el caso de persistencia del flujo y crecimiento del aneurisma (Figura 4)(10-12).

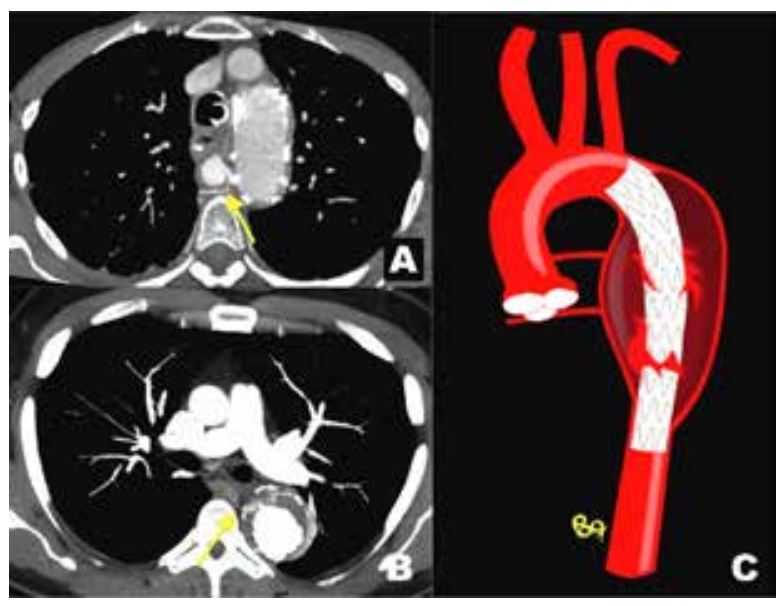

Figura 3. Endoleak tipo II. a) y b) Cortes axiales de TC de tórax contrastada de paciente con disección de la aorta torácica descendente tratada con endoprótesis. Presenta fugas de contraste por flujo retrógrado desde arterias intercostales (flechas). c) Esquema representativo.

Figura 1. Endoleak tipo IA. a) Esquema representativo. Reconstrucción sagital oblicua (b) y corte axial (c) de Tomografía computada (TC) de tórax contrastada de paciente con hematoma intramural de la aorta ascendente y disección desde el origen de la arteria subclavia izquierda, tratado con prótesis endovascular en segmento ascendente y descendente. Se identifica salida del medio de contraste (flecha) en relación al extremo proximal de la prótesis vascular. 


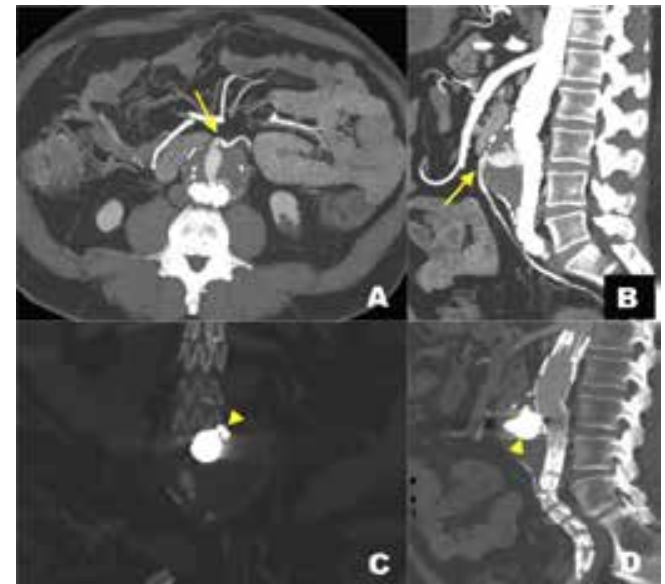

Figura 4. Endoleak tipo II. Corte axial (a) y reconstrucción sagital (b) con proyección de máxima intensidad (MIP) de TC contrastada de paciente con aneurisma de la aorta abdominal infrarrenal tratado con endoprótesis. Presenta una fuga de contraste por flujo retrógrado desde la arteria mesentérica inferior (flechas). Imágenes de reconstrucción coronal (c) y sagital (d) de TC posterior al tratamiento del endoleak con embolización de histocril (cabezas de flecha).

- Endoleak tipo III, se produce una fuga a través de la endoprótesis secundaria a rotura, defecto de la endoprótesis o por falla del sello entre los módulos protésicos (Figura 5) ${ }^{(3,4,9,13)}$. En la angiografía por TC, el contraste se encuentra en contacto directo con la endoprótesis, respetando el saco aneurismático periférico. También en la TC se evalúa la integridad de los elementos metálicos de la endoprótesis. Siendo necesaria la reparación inmediata en caso de estar presente ${ }^{(10,11)}$.

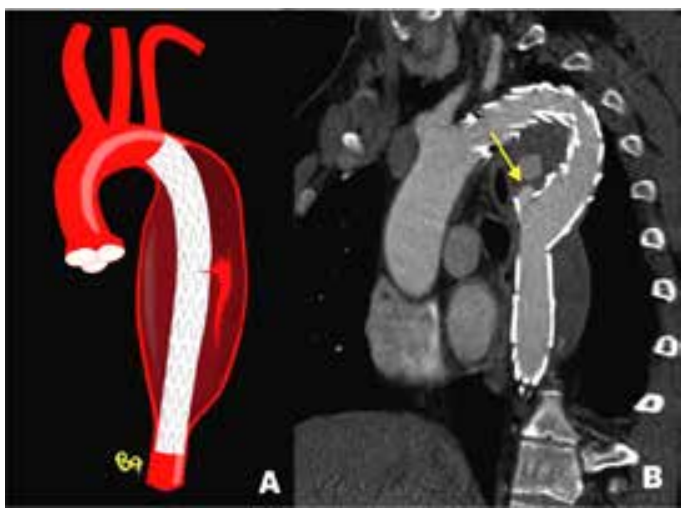

Figura 5. Endoleak tipo III. a) Esquema representativo. b) Reconstrucción sagital oblicua de TC contrastada de paciente con extensa disección de la aorta torácica descendente que fue reparada con 2 módulos de prótesis endovascular. Se identifica una pérdida de continuidad de la prótesis vascular en el sitio de unión de ambos módulos, lo que genera salida del contraste endovenoso (flecha).

- Endoleak tipo $I V$, se genera por una porosidad de la endoprótesis, asociado a anticoagulación intensa del paciente. Habitualmente se detecta en la angiografía inmediatamente tras la instalación de la endoprótesis y se manifiesta como la salida de contraste a través de la endoprótesis, sin identificarse un defecto estructural (Figura 6) ${ }^{(3,4,9,10)}$. El diagnóstico de esta endofuga es de descarte y se corrige una vez ajustada la anticoagulación ${ }^{(4,10)}$. Actualmente se cree que este tipo de endoleak ha disminuido debido a la mejoría en la calidad de los materiales protésicos, siendo éstos más herméticos.

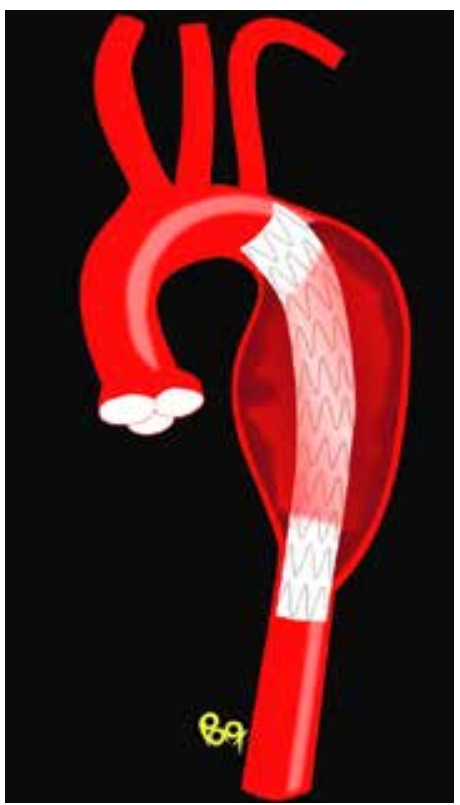

Figura 6. Endoleak tipo IV. Esquema representativo.

- Endoleak tipo $V$, se define como una expansión aneurismática, sin una fuga identificable de contraste endovenoso hacia el aneurisma en la TC, luego de una reparación endovascular exitosa (Figura 7). Se produce por la tensión que ejerce la presión sanguínea sobre la endoprótesis, la que es transmitida al saco aneurismático, resultando en una dilatación de éste. También se plantea que puede corresponder a la manifestación de una fuga no visualizada con las técnicas de imágenes actuales ${ }^{(4,7,9,13)}$.

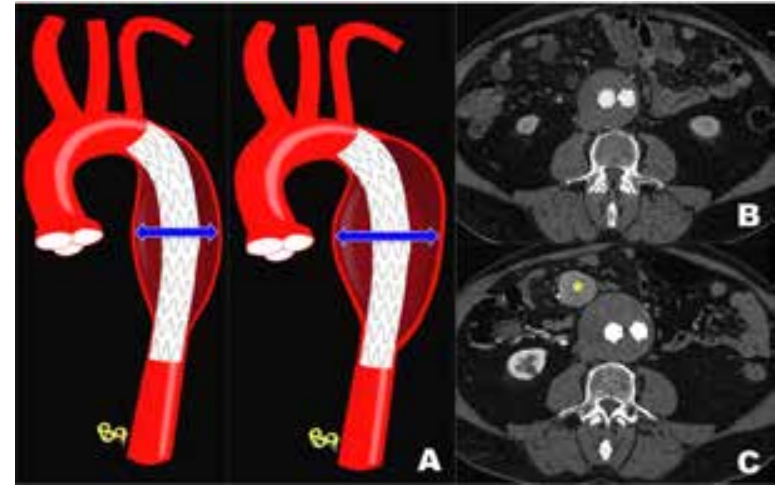

Figura 7. Endoleak tipo V. a) Esquema representativo del crecimiento del saco aneurismático en el tiempo, sin evidencias de endoleak. b) Cortes axiales de TC contrastada de paciente con aneurisma de la aorta abdominal infrarrenal tratado con endoprótesis. c) Control a los 2 años que demuestra aumento de $1,5 \mathrm{~cm}$ del diámetro del saco aneurismático, sin causa aparente. Nótese una adenopatía mesentérica hiperdensa a la derecha del saco aneurismático, que resultó corresponder a metástasis de carcinoide ileal (asterisco). 


\section{Conclusión}

Los médicos involucrados en el diagnóstico, tratamiento y control de pacientes con patología aórtica aneurismática deben conocer las manifestaciones imaginológicas de las complicaciones del manejo endovascular, en especial de los endoleak, considerando el aumento en la prevalencia de la enfermedad ateroesclerótica y como consecuencia, de la patología aneurismática aórtica.

La tomografía computada contrastada es el método de elección para la evaluación periódica de las endofugas. Dentro del protocolo de evaluación se sugiere la realización de una fase sin contraste y una fase venosa o tardía, ya que permiten identificar fugas no claramente evidentes en la fase arterial.

Para el radiólogo es imprescindible conocer, identificar oportunamente y ser capaz de clasificar los endoleaks o endofugas, ya que éstas pueden determinar una elevada morbilidad y mortalidad si no son detectadas y tratadas a tiempo.

\section{Bibliografía}

1. Stolzmann P, Frauenfelder T, Pfammatter T, Peter $N$, Scheffel $H$, Lachat $M$ et al. "Endoleaks after Endovascular Abdominal Aortic Aneurysm Repair: Detection with Dual-Energy Dual-Source CT". Radiology 2008; 249(2): 682-691.

2. Mita T, Arita T, Matsunaga N, Furukawa M, Zempo $\mathrm{N}$ et al. "Complications of endovascular repair for thoracic and abdominal aortic aneurysm: An imaging Spectrum". RadioGraphics 2000; 20: 1263-1278.

3. Yu Tongfu, Zhu Xiaomei, Tang Lijun, Wang Dehang, Saad Nael. "Review of CT Angiography of Aorta". Radiol Clin Am 2007; 45: 461-483.

4. White G, May J, Waugh R, Chaufour X, Yu W. "Type III and type IV endoleak: toward a complete defini- tion of blood flow in the sac afeter endoluminal AAA repair." J Endovasc Surg 1998; 5: 305-309.

5. Lezzi R, Cotroneo A, Filippone A, Di Fabio F, Quinto F, Colosimo C. et al. "Multidetector CT in Abdominal Aortic Aneurysm Treated with Endovascular Repair: Are Unenhanced and Delayed Phase Enhanced Images Effective for Endoleak Detection? " Radiology 2006; 241(3): 915-921.

6. Sommer W, Becker C, Haack M, Rubin G, Weidenhagen $\mathrm{R}$, Nikolaou K, et al. "Time-resolved CT angiography for the Detection and classification of endoleaks." Radiology 2012; 263(3): 917-926.

7. Bastos R, Filho A, Blasbalg R, Caffaro R, Karakhanian W, Rocha A. "A multidetector tomography protocol for follow-up of endovascular aortic aneurysm repair". Clinics 2011; 66(12): 2025-2029.

8. White G, Yu W, May J. "Endoleak" A Proposed New Terminology to Describe Incomplete Aneurysm Exclusion by an Endoluminal Graft. J Endovasc Surg 1996; 3: 124-125.

9. Bashir M, Ferral H, Jacobs C, McCarthy W, Goldin M. "Endoleaks After Endovascular Abdominal Aortic Aneurysm Repair: Management Strategies According to CT Findings." Am J of Roentgenol 2009; 192(4): 178-186.

10. Agarwal $P$, Chughtai $A$, Matzinger $F$, Kazerooni E. "Multidetector CT of Thoracic Aortic Aneurysms". RadioGraphics 2009; 29 (2): 537-552.

11. Hong C, Heiken J, Sicard G, Pilgram T, Bae K. "Clinical Significance of Endoleak Detected on Follow-Up CT After Endovascular Repair of Abdominal Aortic Aneurysm". Am J of Roentgenol 2008; 191: 808-813.

12. Shah A, Stravropoulos W. "Imaging Surveillance following Endovascular Aneurysm Repair ". Seminars in interventional radiology 2009; 26(1): 10-16.

13. Bucci F, Fiengo L, Valerio N, Ferdani M. "Late type IIIb endoleak after endovascular aneurysm repair: case report and review of the literature". G Chir 2011; 32(6): 329-333. 